\title{
DESARROLlO DE La TEORÍa ORGaNizaCiONAL, EN TORNO A LAS DINÁMICAS DEL CAPITALISMO Y LA INNOVACIÓN*
}

\author{
Development of organizational theory, \\ around the dynamics of capitalism and innovation
}

\author{
Yesid Ariza ${ }^{1}$
}

Recibido: Enero 29 de 2016/Aceptado: Mayo 5 de 2016

\section{RESUMEN}

Este documento pretende exponer el pensamiento sobre la dirección de la Teoría Organizacional, en términos de Innovación a partir de las dinámicas del Capitalismo.

El documento arranca mostrando unos recorridos sobre diferentes estadios de las diferentes escuelas y teorías que sobre las organizaciones se han desarrollado y las contribuciones que éstas han aportado al estudio de la organización desde un punto de vista completo. Se muestra el desarrollo de la función administrativa desde la Escuela Clásica hasta el Paradigma Competitivo, pues este último tiene como clave la innovación. Asimismo, se muestra los hitos del capitalismo y su influencia en las corrientes de pensamiento administrativo.

La fundamentación teórica tiene que ver con el concepto de Innovación, desde los clásicos hasta las posturas más contemporáneas, desde la necesidad de Innovar hasta la Innovación por estrategia. Al final se invita al lector a discutir como estrategias innovadoras robustecerían la Teoría Organizacional.

Palabras clave: Teoría Organizacional, Innovación, Capitalismo, Economía.

\section{ABSTRACT}

This document aims to expose the thinking on the direction of the Organizational Theory, in terms of Innovation from the dynamics of Capitalism.

The document starts showing some routes on different stages of the different schools and theories that have been developed about the organizations and the contributions that these have contributed to the study of the organization from a complete point of view. It shows the development of the administrative function from the Classical School to the Competitive Paradigm, since the latter has the key to innovation. It also shows the milestones of capitalism and its influence on the currents of administrative thought.

The theoretical foundation has to do with the concept of Innovation, from the classics to the most contemporary positions, from the need to Innovate to Innovation by strategy. At the end the reader is invited to discuss how innovative strategies would strengthen the Organizational Theory.

Keywords: Organizational Theory, Innovation, Capitalism, Economy.

Cómo referenciar este artículo: Ariza, Y. (2016). Desarrollo de la teoría organizacional, en torno a las dinámicas del capitalismo y la innovación. Ad-Gnosis, 5(5), 141-150.

* Ensayo reflexivo derivado de revisión de literatura.

1. Profesor Universitario y Consultor Empresarial. Magíster en Administración de Empresas e Innovación. Doctotrante en Administración de Empresas. Profesor, Corporación Unificada Nacional. yesid_ariza@cun.edu.co 


\section{Introducción}

\section{Desarrollo de la Función Administrativa}

Existen distintas formas de pensar sobre la Administración como disciplina; Fayol la vio como una de las funciones de la empresa junto a las de operación, finanzas, mercadeo y gestión humana; hay que recordar que Fayol (1990) dividió las operaciones industriales y comerciales en seis grupos: Funciones Técnicas: Relacionadas con la producción de bienes o de servicios de la empresa; Funciones Comerciales: Relacionadas con la compra, venta e intercambio; Funciones Financieras: Relacionadas con la búsqueda y gerencia de capitales; Funciones de Seguridad: Relacionadas con la protección y preservación de los bienes de las personas; Funciones Contables: Relacionadas con los inventarios, registros, balances, costos y estadísticas; Funciones Administrativas: Relacionadas con la integración de las otras cinco funciones. Las funciones administrativas coordinan y sincronizan las demás funciones de la empresa, siempre encima de ellas.

Algunos otros autores, como Drucker (1954), la consideraron como una profesión, otros como Mintzberg (2009), la trataron como un oficio. Lo importante es que los conocimientos, teorías y escuelas de pensamiento que se han venido acumulando desde el inicio de la Administración hacen que tenga un acervo interesante y una fundamentación teórica robusta, asimismo, es importante mencionar que la Administración ha influido en otras áreas del conocimiento como la ingeniería, la pedagogía y la política.
Por otro lado, Max Weber, sociólogo y filósofo alemán, considerado como uno de los autores más profundos del siglo $\mathrm{XX}$, es citado para incluir parte de su obra en la Administración; relacionada con la burocracia se ha incluido en el enfoque estructuralista de la Administración, mostrando su obra como una manera de organizar y dominar (Wren, 1979; Minztberg, 1979; Koontz, O’Donnell \& Weilhrich, 1984; Miller, 1985), apoyando otros postulados también significativos, por ejemplo: los aspectos psicológicos y sociológicos que entran en juego en la administración de una empresa.

El objeto de la Administración, como disciplina, es el desarrollo de procesos a través de los cuales las empresas logren resultados en su operación; la Administración se ha considerado como parte integral del área de las ciencias económicas y en el campo más amplio de las ciencias sociales, pero se alimenta de un alto número de disciplinas entre las cuales se destacan la Psicología, la Sociología, la Estadística y por supuesto la Economía. Por todo lo anterior, se puede concluir que la Administración es una disciplina de acción.

La Administración como disciplina independiente se inicia a comienzos del siglo pasado y su devenir se puede trazar según las siguientes etapas de cambio: escuela clásica, escuela humanista, teoría situacional, gerencia estratégica y gestión competitiva.

La Escuela Clásica: En esta fase se inicia el discurso administrativo, se establecen los funda- 
mentos de la administración como disciplina relativamente autónoma con los significativos aportes de Taylor y Fayol (1990). El primero se dedicó a estudiar el trabajo metódicamente en lo que se denominó luego el estudio de tiempos y movimientos y estableció el "principio" de separación entre la realización del trabajo y la planeación del mismo. Pero el aporte significativo en el campo disciplinar lo hizo realmente Fayol, quien estableció por primera vez las áreas de la empresa: producción, ventas, finanzas y personal y, sobre todo, definió las funciones administrativas, estableciendo como tales a la planeación, la organización, la coordinación, el mando y el control, entendía estas funciones como las actividades generales que debía desarrollar todo administrador.

En relación con la Escuela Humanista: Una pléyade de científicos sociales, especialmente psicólogos, comenzaron a explorar las implicaciones humanas de la gestión. El desarrollo se inicia con los experimentos de Elton Mayo* quien examinó la relación entre la iluminación y la productividad del trabajo. También en esta escuela fue importante el aporte teórico de McGregor quien propuso su conocido modelo de teorías $\mathrm{X}$ y $\mathrm{Y}$, en el cual se definían dos estilos de gestión: el $X$, fundamentalmente autoritario y el $Y$, de carácter participativo. De la misma manera, por esta época se dedicó

* Elton Mayo llevo a cabo un experimento en Western Electric Company que tenía como objetivo determinar la relación entre la satisfacción del hombre y la eficiencia de los obreros en la producción. Se llamó el Experimento de Hawthorne. esfuerzo al tema de la motivación en el trabajo, en el cual se destacó el aporte de Maslow (2005), con su famosa escala de necesidades: básicas, de seguridad, sociales, egoístas y de autorrealización, esta última relacionada por este autor con la creatividad. En la misma dirección, Hetzberg (2010), planteó su clasificación de los incentivos en dos tipos: de higiene o exógeno y motivadores o endógenos, estos últimos fueron resultados que luego aparecerían en la posición acerca de la creatividad de Amabile, por la vía de la motivación intrínseca.

La Teoría Situacional: Se basa en el enfoque de sistema abierto. La idea que se tenía era que la empresa es un sistema abierto y la gestión busca adaptar la empresa al contexto. Así que dependiendo del estado del entorno, se tendrían que aplicar determinados tipos de gestión. Una segunda teoría situacional la plantearon Burns y Stalker (1961), ellos sostuvieron que el tipo de estructura que una empresa emplee depende del estado del entorno donde ella se ubique. Finalmente se debe tomar en cuenta la teoría del liderazgo de Fiedler** quien propuso que el estilo de dirección que emplee un determinado dirigente depende de la situación en la cual se desarrolle su liderazgo.

Bajo la presión del enfoque de sistema abierto, en los años 60 del siglo pasado, aparece la

** A principios de 1951, Fred Fiedler desarrolló el primer Modelo de Contingencia para el Liderazgo, a partir de la relación entre el rendimiento organizacional y las actitudes del Líder. 
planeación estratégica que se polariza frente a la planeación operativa tradicional por aquella época, y se basaba en dos tesis centrales: que se debe planear la empresa como totalidad, no como una serie de áreas dispersas o especializadas áreas empresariales de Fayol, y que la empresa debe responder al estado del entorno. Aparece así el proceso de planeación estratégica consistente en estudiar el entorno, examinar lo interno para, con base en ellos, determinar luego los objetivos y por último la estrategia. Autores que se destacaron en esta fase fueron Steiner, Andrews y Ansoff (1990). Este último, uno de los teóricos más creativos, introdujo una clasificación de las estrategias de crecimiento que se ha mantenido hasta hoy. Este mismo autor dio el salto al concepto de gerencia estratégica extendiendo la aplicación de la planeación a la estructura, el liderazgo, el control y la cultura organizacional.

Aunque el tema de la Competitividad es antiguo en Economía, especialmente por el estudio de los mercados (de competencia perfecta o monopolística), su aplicación en grande es más bien reciente. El primer modelo competitivo lo planteó Ohmae (2004), quien decía él que los actores claves de la competitividad eran: la compañía, el cliente y los competidores (modelo de las CCC).

En el comienzo de los 80, Porter (1990), lanza su modelo de fuerzas competitivas de la industria en el cual se adicionan nuevos actores de la competitividad. El modelo incluye a los rivales y los consumidores como lo hacía Ohmae
(2004), pero adiciona los proveedores, los entrantes y los sustitutos. Aparece posteriormente el modelo de recursos y capacidades que destaca los recursos de la empresa sobre todo en lo que tiene que ver con sus características distintivas para la competitividad. Unos autores que dan un giro desde las actividades hacia las habilidades fueron Hamel y Prahalad (1996), quienes introdujeron el concepto de competencias centrales como el conjunto de conocimientos y técnicas que le permiten a una empresa competir en el mercado.

Aparece posteriormente el modelo de recursos y capacidades que destaca los recursos de la empresa sobre todo en lo que tiene que ver con sus características distintivas para la competitividad. Unos autores que dan un giro desde las actividades hacia las habilidades fueron Hamel y Prahalad (1996), quienes introdujeron el concepto de competencias centrales como el conjunto de conocimientos y técnicas que le permiten a una empresa competir en el mercado.

Un producto importante del desarrollo competitivo es la aparición de los sistemas empresariales de gestión, entre los cuales se destacan el de gestión de calidad, servicio al cliente y gerencia innovadora. Este último se basa en una estrategia de diferenciación concentrada en el cambio radical de productos, servicios y procesos, sustentado por el recurso humano innovador, que se fundamenta en la creatividad. Es bueno destacar también a Drucker (1985), uno de los más grandes de la geren- 
cia, quien abordó el tema de las estrategias innovadora y las fuentes de la innovación. Así mismo hay un autor más reciente pero que está en la línea innovadora, se trata de Hamel (2012), quien defiende la innovación radical.

\section{Dinámicas del Capitalismo}

La revolución económica que muestra Heilbrouner (1985), tiene las siguientes características:

- La economía se mostró como una fuerza para sobrevivir, después de la acumulación de cosechas, Esclavitud, etc. La economía muestra la posibilidad de sobrevivir sin estar bajo el mando de otro hombre.

- La economía pasa por periodos de analfabetismo total (Francia, año 1300), rudimentario registro de operaciones, pero con compresión de entorno (Alemania, año $1500)$, inicios de entendimiento de relaciones de mercado (Boston, año 1666).

- Después Francia, Inglaterra y otros países muestran el desarrollo de la estructura económica y termina conociéndose los postulados "el hombre busca, naturalmente, un lucro", "no hay leyes que puedan matar el afán de ganancia", "la ganancia es el centro del círculo del comercio".

- Se crea un nuevo concepto "el hombre económico", esta entidad deambula por senderos sinuosos, buscando ganancia.

Las cantidades, los precios, las autorregulaciones, muestran la obra de Smith completa y preocupada por la dinámica entera de la sociedad, dentro de la cual está el mercado, ese ente en el que todo sucede, con leyes, por ejemplo, la de acumulación, que hacen comprender la riqueza como algo más allá del dinero, que se compra con este, pero es útil para la sociedad. Además de estos conceptos, la aparición de la empresa en el siglo XVIII y las condiciones sociales y económicas de esa época, muestra, el sistema de producción que comenzaba a aparecer. Se habla, entonces de rupturas que se dieron en el paso del trabajo tradicionalmente a mano al trabajo moderno de la época; estas rupturas fueron: separación del campo y la ciudad, imposición de forma de usar las herramientas, rompimiento con la naturaleza, separación del productor con el poseedor. Estas rupturas crearon una cultura industrial. La recién cultura industrial, se robusteció con los principios de división y especialización del trabajo (Higuera, 2013).

Se siguió desarrollando el modelo capitalista y la economía pasó de un modelo Keynesiano en el cual había control del estado en los precios y la calidad del producto ofertado, a un auto control con características descabelladas; las multinacionales muestran solo la flecha dorada del sistema, que es el consumo, pero la extracción que es un eufemismo, pues la realidad es la destrucción y deslocalización de costos y la colocación que es otro eufemismo, pues la verdad es un impune vertimiento de desperdicios y súper tóxicos, están fuera de foco.

Después de la Segunda Guerra Mundial el modelo cambio de producción a consumo y las empresas, con su avanzada de información, ha 
corroído las mentes de las personas, básicamente con dos estrategias, que al final puede ser la misma que se complementa asimisma:

La obsolescencia programada, que consiste en reducir a propósito la vida útil de los productos, pero de manera tal que los consumidores no lo consideren injusto; la otra es la obsolescencia percibida, que consiste en hacer sentir fuera de sitio a las personas que no contribuyen al consumo, mediante la publicidad y la infoxicación, así también el ambiente obeso génico.

Los gobiernos dependen y defienden las multinacionales y sus prácticas devastadoras; el mundo no está bien administrado y no puede seguirse explotando un modelo lineal en un planeta finito, la alternativa es el cambio de sistema de producción: uno circular o producción verde, o simplemente, solo sea pasar de How to make money a How to make better.

\section{Fundamentación Teórica}

\section{La Innovación}

Para acercarse al concepto de innovación, es preciso hacer un recorrido por diferentes autores y disciplinas, pues este término es interdisciplinario y tiene aristas profundas en ambientes económicos, competitivos, ambientales, etc. Es así como se construye el concepto contextualizado de innovación para efectos de este trabajo de grado.

Schumpeter (1934) trabajo el concepto de "destrucción creativa" para dar a conocer su pensamiento sobre la forma como la innova- ción radical podría cambiar el rumbo de la economía de las naciones mientras que la innovación progresiva, como solía llamarla, alimenta el proceso del cambio que se evidencia. El autor hace un aporte significativo al clasificar los tipos de innovación, así:

- Introducción de nuevos productos.

- Introducción de nuevos métodos de producción.

- Apertura de nuevos mercados.

- Desarrollo de nuevas fuentes de suministro.

- Creación de nuevas estructuras de mercado.

Asimismo, el Manual de Oslo (2005) hace énfasis en la introducción con relativo éxito al mercado de los aspectos contemplados en la definición referenciada anteriormente, esto deja entrever que es necesario un método de medición de la percepción del entorno, respecto a la innovación de esta empresa.

En 1985 Peter Drucker en su libro Innovation and Entrepreneurship, argumenta que:

La innovación es la herramienta específica de los empresarios innovadores; el medio por el cual explotar el cambio como una oportunidad para un negocio diferente. Es la acción de dotar a los recursos con una nueva capacidad de producir riqueza. La innovación crea un 'recurso'. No existe tal cosa hasta que el hombre encuentra la aplicación de algo natural y entonces lo dota de valor económico. (Drucker, 1985, p.98) 
Después de analizar y contrastar las diferentes definiciones de innovación, se propone la siguiente: Es la capacidad del ser humano, equipo u organización, de introducir al mercado de forma exitosa, una mejora o una nueva idea de producto, negocio o concepto.

\section{Tipos de Innovación}

Los diferentes autores han coincidido en clasificar la innovación desde dos puntos de vista: El primero tiene que ver con la Naturaleza del Cambio; es decir, el asunto mismo que se cambiará, en este sentido se refieren al objeto de investigación y desarrollo. El otro punto de vista es el Grado del Cambio; dicho de otra manera, es la velocidad o el impacto producido por los resultados en relación con el nivel de diferenciación que se obtiene después de la aplicación del proceso de innovación.

Mendoza (2006), identifica cuatro tipos de innovación, de negocio, producto, proceso y administrativa, esto en relación con la naturaleza del cambio; respecto al grado de cambio, pueden considerarse dos tipos de innovación, la incremental y la radical.

La innovación radical tiene que ver con el efecto que causa en las capacidades de la organización, es decir, si el nuevo producto, negocio, concepto o mercado rompe de golpe con la cultura establecida de consumo o de negocios o de actuación. El resultado de la innovación radical afecta, positiva o negativamente, al entorno y al ambiente de la empresa; es posible que esa afectación pueda ser negativa cuando las capacidades existentes no sean suficientes para manejar el nuevo conocimiento; expresado en otra forma, es posible que la empresa que generó el cambio tenga problemas para seguir produciéndolo.

La Innovación Radical, entonces, es el cambio abrupto en cualquier tipo de Innovación, efectuado por una empresa con el fin de impactar positivamente en su entorno y su ambiente.

El término innovación disruptiva fue introducido por Clayton Christensen, profesor de la Harvard Business School, para hacer una diferenciación de la incremental. Para este autor, considerado el gurú de la innovación disruptiva, el problema que tenía la innovación incremental es que solía traer consigo una importante alza de los costos y una progresiva disminución de la percepción de incremento de valor por parte del cliente. Ante ello, Christensen propone la innovación disruptiva como una buena estrategia que trae consigo una importante mejora en la percepción de valor por parte del mercado.

De acuerdo a Christensen (1997), quien opina que las innovaciones pueden apreciarse a lo largo de un continuom, esto quiere decir, que parten desde un punto evolutivo y llega hasta lo revolucionario; ahora bien, hay que entender que las innovaciones evolutivas son esencialmente importantes para mantener y ampliar las cuotas de mercado en los mercados existentes, mientras que las innovaciones revolucionarias son el epicentro de la creación de riqueza. 
Para Christensen (1997); las innovaciones revolucionarias son la base de las industrias, productos, tecnologías y servicios del mañana. La expresión innovación disruptiva se ha aplicado para entender aquellas innovaciones que son de naturaleza extremadamente revolucionaria o discontinuas, y que llevan a los clientes y consumidores a adoptar nuevas formas de pensar, actuar y consumir descartando los modelos ya existentes (Christensen \& Rosenbloom, 1995; Hamel, 2002).

Para este documento se propone la siguiente definición para Innovación Disruptiva: La innovación disruptiva es aquella que crea una singularidad* en la operación de la empresa, a partir del aporte conjunto de los elementos de las dimensiones Humana, Técnica y Organizacional inmersas en la realidad empresarial para desarrollar productos que diferencien a la empresa en un mercado que no se había contemplado.

\section{Metodología}

Se realizó una selección de múltiples trabajos publicados; tanto artículos como libros en relación con el tema de Teorías de la Administración, Teoría Organizacional, Innovación, Economía y Capitalismo. Se utilizó las siguientes palabras clave: Teoría Organizacional, Innovación, Capitalismo, Economía.

Después del proceso de selección, a juicio del autor de este documento, se procedió con un

* Distinción o separación de lo común (Diccionario de la Real Academia de la Lengua Española). estudio exploratorio sobre los resultados de las investigaciones de los diferentes autores, asimismo, de las diferentes metodologías utilizadas.

La redacción del documento se basó en una reseña sobre los hallazgos encontrados y la postura del autor sobre las posiciones de los diferentes autores.

\section{Resultados y discusión}

La Teoría Organizacional toma como base de conocimiento la Administración de Empresas, y luego se enmarca en el paradigma de la competitividad para concentrarse, dentro de este, en la gerencia innovadora, especialmente en los aspectos de estrategias innovadoras, procesos de innovación, gestión de la innovación, intraemprendimiento, creación de nuevos negocios e innovación tecnológica.

Ha evolucionado desde el enfoque de la Teoría Clásica y las funciones de la empresa, hasta un enfoque contemporáneo con funciones administrativas o áreas de gestión. La tabla de más abajo escenifica el cambio:

Tabla 1. Enfoque clásico y enfoque contemporáneo

\begin{tabular}{|l|l|}
\hline \multicolumn{1}{|c|}{ Enfoque de Fayol } & \multicolumn{1}{c|}{ Enfoque contemporáneo } \\
\hline Función de la Empresa & \multicolumn{1}{c|}{ Funciones Administrativas } \\
\hline Técnicas & Área de Producción u Operaciones \\
\hline Comerciales & Área de Ventas o Marketing \\
\hline De seguridad & $\begin{array}{l}\text { Área de Gestión de Personal y otras } \\
\text { pasaron a un nivel inferior }\end{array}$ \\
\hline Contables & Se subordinaron a las financieras \\
\hline Financieras & Área Financiera \\
\hline Administrativas & Área Gerencial y Estratégica \\
\hline
\end{tabular}

Fuente: Elaboración propia 
La obra de Weber (1958) es completa y toca además de la economía, la sociología, la psicología y por supuesto la administración; en este periplo por las diferentes disciplinas, Weber dejar ver la manera como debe estructurarse la empresa, de manera que la armonía social, del sistema social concreto, permee la realidad abstracta de la empresa, representada por los cargos, las relaciones y las funciones. A pesar de la Jaula de Hierro, el proceso estructuralista de Weber apoya la innovación como disciplina especializada en competitividad y hoy día el desarrollo de la innovación se muestra articulado, robusto y concreto, precisamente, por la especialización.

La Teoría Organizacional ha sufrido cambios y ajustes que llevan el estudio de la organización desde el oficio, como se empezó con la nueva cultura industrial al inicio de la industria, producto de la primera revolución industrial; hasta el manejo del entorno y creación de escenarios, usando la prospectiva en el paradigma de la competitividad. Las escuelas, las teorías, los autores y la realidad propia de las organizaciones en la sociedad se van mostrando más inclinadas a la revolución del mercado, como ente vivo, mas que como lugar virtual de desarrollo de relaciones entre actores y dinámicas de oferta y demanda. La innovación se muestra como llave de la competitividad, paradigma en el que se encuentra inmersa a organización.

Los gerentes deben aceptar el reto de innovar en la gestión empresarial porque es la manera de crecer económicamente y de ser competitivo (Hammel, 2012). Ante la anterior premisa la nueva actitud gerencial se debe orientar al estímulo personal de la innovación, de manera que se convierta en un proceso complejo, enmarcado por un sistema completo de creatividad e innovación en las empresas. La realización de las ideas es un proceso concreto, debe ejecutarse con compromiso, dedicación y debe ser una decisión estratégica de las organizaciones, por ejemplo:

- Diseñar modelos de innovación.

- Entrenamiento de empleados de acuerdo a sus aptitudes.

- Diseño e implementación de sistemas de comunicación formales para el estimulo a nuevas ideas.

- Medición de los resultados de las nuevas ideas.

- Creación de equipos de trabajo efectivos en negociación, creatividad e innovación.

- Culturización en filosofía de calidad, entendiéndose calidad como la decisión de desempeñarse con eficiencia y eficacia.

\section{Referencias}

Burns, T. \& Stalker, G. M. (1961). Organizaciones Mecanicistas y Orgánicas. Harvard Bussines School Press.

Christensen, C. M. (1997). The Innovators Dilemma: when new technologies cause great firms to fail. Boston, Massachusetts: Harvard Business School Press.

Christensen, C. M. \& Rosenbloom, R. (1995). Explaining the attacker's advantage: technological paradigms, organizational dynamics and the value network. Research Policy, 24, 233-257. 
Drucker, P. (1954). La Práctica del Management.

Drucker, P. (1985). La innovación y el empresario innovador. Ed. Edhasa.

Hamel, G. (2002). Leading the Revolution: How to Thrive in Turbulent Times by Making Innovation a Way of Life. Boston: Harvard University Press.

Hamel, G. (2012). Lo que importa ahora. Bogotá: Grupo Norma.

Hamel, G. \& Prahalad, C.K. (1996). Compitiendo para el futuro. Harvard Bussines School Press.

Heilbrouner, R. (1985). Vida y obra de los grandes economistas. Barcelona: Orbis.

Hetzberg, F. (2010). The Motivation to Work. Library of Congress.

Higuera Ojito, V. (2013). Análisis económico de la demanda educativa en programas de Ciencias Económicas: un modelo de elección basado en un logit binomial. Ad-Gnosis, 2(2), 13-21.

Koontz, H., O'Donnell, C. \& Weihrich, H. (1984). Management. New York: McGraw-Hill.

Manual de Olso (2005). Guidelines for Collecting and Interpreting Innovation data. 3 Ed.

Maslow, A. (2005). El Management Según Maslow: Una Visión Humanista para la Empresa de Hoy. Barcelona: Editorial Paidós.
Mendoza, J. M. (2006). Innovación por lo alto, Asesores del 2000. Factores claves identificados en las alianzas Universidad-Industria en proyectos de innovación ejecutados en la costa Caribe colombiana. Abello Llanos Raimundo Ph.D. 2007, p.34.

Miller, R. (1985). La direction des entreprises. Concepts et aplications. Montreal Agence D'art.

Mintzberg, H. (2009). Managin. Bogotá: Norma.

Mintzberg, H. (1979). THe structuring of organization. Englewood Clif, New Jersey: Prentice-Hall.

Ohmae, K. (2004). La Mente del Estratega. España: McGraw-Hill.

Porter, M. (1990). The Competitive Advantage of Nations. Londres: MacMillan.

Schumpeter (1934). Economic Reconstruction: The Columbia Report. Journal of political economic. The Universitu of Chicago Press.

Weber, M. (1958). The Spirit of capitalism. In The Protestant Ethic and the Spirit of Capitalism (traducción de T. Parsons) (pp. 47-79). New York: Charles Scribner's Sons.

Wren, D. A. (1979). The evolution of management tought. $2^{\text {a }}$ ed. New York: Jonh Wiley and Sons. 